\title{
Proportionate Target Population Estimates Used by National Immunization Programmes in Sub-Saharan Africa and Comparison with Values from an External Source
}

\author{
David W. Brown ${ }^{1}$, Anthony H. Burton ${ }^{2}$, Marta Gacic Dobo², Richard Mihigo ${ }^{3}$ \\ ${ }^{1}$ United Nations Children's Fund, New York, USA \\ ${ }^{2}$ The World Health Organization, Geneva, Switzerland \\ ${ }^{3}$ The World Health Organization Regional Office for Africa, Brazzaville, Republic of Congo \\ Email: dbrown@unicef.org
}

Received 15 June 2014; revised 14 July 2014; accepted 13 August 2014

Copyright (C) 2014 by authors and Scientific Research Publishing Inc.

This work is licensed under the Creative Commons Attribution International License (CC BY). http://creativecommons.org/licenses/by/4.0/

(c) (i) Open Access

\section{Abstract}

Background: In order to effectively plan the delivery of immunization services, manage stock and supply levels and target interventions, national immunization programmes (NIP) must have an estimate of the target population they serve. To overcome challenges with target population estimation, some NIPs apply "rule-of-thumb" conversion factors to total population estimates. We compare these proportionate target population values with those from an external source. Methods: Using data reported by national immunization programmes in sub-Saharan Africa, we computed the proportionate target population as the number of births, surviving infants and children under 5 years of age, respectively, as a proportion of the total population size. We compared these values with those estimates computed from United Nations Population Division (UNPD) data. We then recomputed NIP target population sizes using the proportionate target population values from the UNPD applied to the total population size reported by NIP. Results: Data were available from 47 sub-Saharan Africa countries. Births as a proportion of the total population were greater within reports from NIP (median, 0.0400; IQR: 0.350 - 0.0437) compared to values from UNPD estimates (median, 0.0364; IQR: 0.0332 - 0.0406). Similar patterns were observed for surviving infants (median: NIP, 0.0360; UNPD, 0.0337) and children under 5 years of age (median: NIP, 0.1735; UNPD, 0.1594). The percent difference in proportionate target population ratios between reports from NIPs and the UNPD was $>\mathbf{1 0} \%$ in 23 countries for births, in 18 countries for surviving infants,

\footnotetext{
"Corresponding author.
} 
in 15 countries for children under 5 years of age. After re-computing target populations using UNPD proportionate target population values applied to NIP reported total population, recomputed administrative coverage levels for the third dose of DTP containing vaccine were higher in 32 of the 47 countries compared to reported administrative coverage levels. Conclusion: Because childhood immunization-related target populations are among the more difficult ones to accurately estimate and project, immunization programmes in sub-Saharan Africa are encouraged to include a critical assessment of the target population values, in conjunction with their national statistics system, as part of the on-going programme monitoring process.

\section{Keywords}

Population, Population Estimates, Target Population, Immunization, Immunization Coverage

\section{Introduction}

National immunization programmes are responsible for protecting children from vaccine preventable diseases. To effectively plan the delivery of immunization services, manage stock and supply levels and target interventions, programme managers must have an estimate of the target population they serve, which in most instances consists of the number of births, surviving infants and children under 5 years of age. However, accurate estimates for these population groups are extremely difficult to obtain in many settings, particularly in countries where the last census was more than 10 years ago (i.e., Angola, Central African Republic, Comoros, Democratic Republic of Congo, Equatorial Guinea, Eritrea, Madagascar, Somalia, Uganda) [1].

To overcome this challenge, some national immunization programmes apply "rule-of-thumb" conversion factors to total population estimates, which are relatively more accurate than subnational or age-specific estimates, to obtain an estimated number of births, surviving infants and children under 5 years. These rule-of-thumb conversion factors represent proportionate target population values. The proportionate target population is the number of births (or surviving infants or children under 5 years) as a proportion of the total population size. A quick review of proportionate target population values computed from United Nations Population Division (UNPD) estimates and projections for all countries and territories as well as for sub-Saharan Africa highlight differences in magnitude and declines since the beginning of the Expanded Programme on Immunization in 1974 (Figure 1).

In this report we examine and describe proportionate target population values computed from national-level population data for 2013 reported by national immunization programmes in sub-Saharan Africa. We compared the values based on nationally reported data with those computed from UNPD estimates. We also recomputed target population sizes using the national proportionate target population values from the UNPD applied to the total population size reported by national immunization programmes. Finally, we recomputed coverage levels for the third dose of DTP containing vaccine $\left(\mathrm{DTP}_{3}\right)$ to examine the impact of differences in the proportionate target population values for sub-Saharan African countries reporting to the World Health Organization (WHO) and United Nations Children's Fund (UNICEF).

\section{Methods}

\subsection{Immunization Target Population Data and Comparisons with an External Data Source}

Each year WHO and UNICEF jointly collect national-level data on the incidence of selected vaccine-preventable diseases, immunization coverage, recommended immunization schedules, vaccine supply and other information on the structure, policies and performance of national immunization systems through the WHO/UNICEF Joint Reporting Form on Immunization (JRF). (Since 2000, more than 95\% of WHO Member States have reported annually.) As part of this annual reporting exercise, national authorities also report the estimated number of children vaccinated according to administrative data (i.e., reports from health service providers) and the number of children in the national target population for each antigen in the national immunization schedule.

During 2014, countries from the WHO African Region reported the number of live births, surviving infants, 


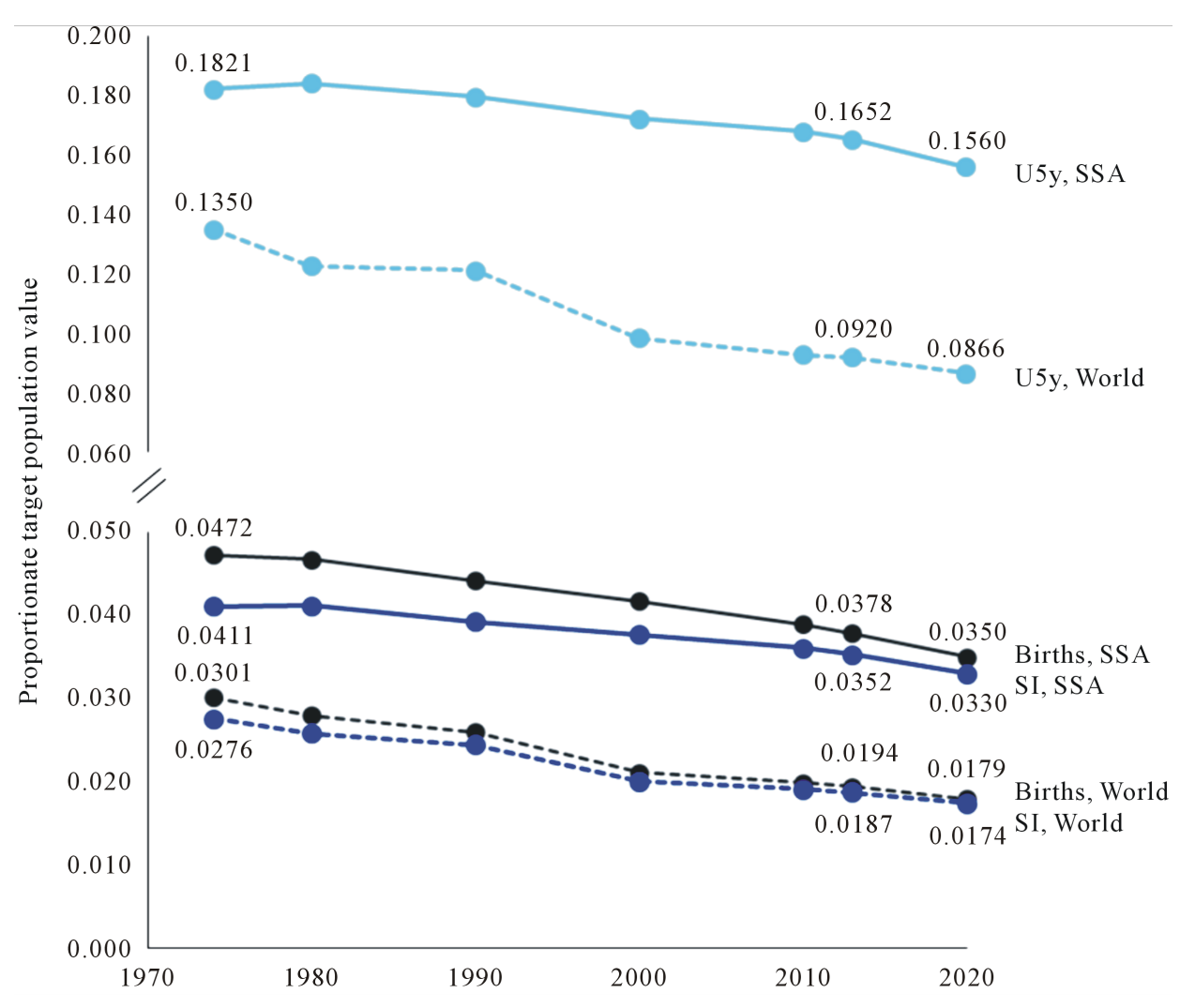

Figure 1. Proportionate target population values for births, surviving infants (SI) and children under 5 years (U5y) computed from UNPD estimates and projections for sub-Saharan Africa (SSA) and all countries, 1974-2020. Data source: United Nations, Department of Economic and Social Affairs, Population Division (2013). World population prospects: The 2012 Revision.

children under 5 years of age and total population size used by the national immunization programme during 2013 on the WHO/UNICEF Joint Reporting Form. The appropriate target population for vaccines administered at birth (e.g., Bacille Calmette-Guérin or BCG; Hepatitis B birth dose) is the estimated number of live births in the country. For other vaccines, the target population most often used for computing coverage is the number of surviving infants. (NB. Some countries use the number of live births as the official denominator for computing coverage levels. Historically, countries with high infant mortality have chosen not to use the number of live births as a target population for vaccines administered after birth since an immunization programme's performance might not be accurately reflected in the presence of high infant mortality even if the programme was vaccinating each and every child that survived.) And, vaccination campaigns (e.g., measles) often target children under 5 years of age.

We abstracted the national number of live births, surviving infants, children under 5 years of age and total population size for the 2013 reporting period as well as the national number of children vaccinated with three doses of DTP containing vaccine $\left(\mathrm{DTP}_{3}\right)$ obtained from national routine administrative data systems. Administrative data are based on aggregated reports from service providers to local health authorities of the number of vaccinations administered during a given period.

We also abstracted the national number of births, surviving infants, children under 5 years of age and total population size from public use files provided by the UNPD [2]. For both the nationally reported population data and that obtained from the UNPD, we divided the number of births by the total population size to obtain births as a proportion of the total population. We repeated this process for surviving infants and children under 5 years. We then computed the percent difference in the national proportionate target population values for births, surviving infants and children under 5 years between the nationally reported values compared to those computed from UNPD data. 


\subsection{Application of Proportionate Target Population and Implications for Coverage}

To examine changes in coverage if one used the UNPD-based national proportionate target population values, we multiplied the total national population size reported by the national immunization programme by the proportionate target population values from the UNPD to obtain a recomputed national number of births, surviving infants and children under 5 years, respectively, for each country. Using the recomputed number of surviving infants as denominator and the nationally reported number of children vaccinated, we recomputed coverage for $\mathrm{DTP}_{3}$. We then compared the recomputed coverage level with the administrative coverage reported by national immunization programmes and describe the observed differences.

\section{Results}

\subsection{Total Population}

Data were available for 47 countries. Sudan, although geographically located in sub-Saharan Africa, reports to the WHO Regional Office for the Eastern Mediterranean rather than the WHO Regional Office for Africa and therefore total population size and the number of children under 5 years was not reported by the Sudan immunization programme to WHO or UNICEF for this exercise. Somalia, geographically located in sub-Saharan Africa reporting to the WHO Regional Office for the Eastern Mediterranean, was an exception to the annual reporting process and data availability for this exercise as a WHO/UNICEF Joint Reporting Form that provided the total population size and number of children under 5 years was submitted by Somalia through UNICEF.

Relative differences in total population reported by national immunization programmes compared to those estimated by UNPD ranged from $0.2 \%$ (Botswana) to 39.7\% (Eritrea) with a median percent difference of $5.7 \%$ (IQR: $2.7 \%-8.2 \%$ ). The percent difference (absolute value) in total population between the two sources was less than 5\% in 22 (47\%) of 47 countries and exceeded 15\% in five countries (Burundi, 20.7\%; Côte d'Ivoire, 17.1\%; Democratic Republic of the Congo, 28.1\%; Eritrea, 39.7\%; Gabon, 20.1\%).

\subsection{Proportionate Target Population: Births, Surviving Infants, Children under 5 Years}

Births as a proportion of the total population based on reports from national immunization programmes ranged from 0.0104 (Mauritius) to 0.0583 (Niger) with a median value of 0.0400 (IQR: 0.0350 - 0.0437) (Table 1). Proportionate target population values for births based on UNPD data ranged from 0.0115 (Mauritius) to 0.0499 (Niger) with a median value of 0.0364 (IQR: 0.0332 - 0.0406).

Surviving infants as a proportion of the total population based on reports from national programmes ranged from 0.0179 (Seychelles) to 0.0536 (Niger) with a median value of 0.0360 (IQR: 0.0328 - 0.0402) (Table 1). Comoros, Mauritius, Senegal and South Africareported the number of births for surviving infants rather than a separate value. Surviving infants as a proportion of the total population per UNPD data ranged from 0.0113 (Mauritius) to 0.0473 (Niger) with a median value of 0.0337 (IQR: $0.0312-0.0379$ ).

Children under 5 years of age as a proportion of the total population based on reports from national programmes ranged from 0.0567 (Mauritius) to 0.2391 (The Gambia) with a median value of 0.1735 (IQR: 0.1514 0.2000) (Table 1). In contrast, values based on UNPD data ranged from 0.0564 (Mauritius) to 0.2072 (Niger) with a median value of 0.1594 (IQR: $0.1470-0.1759$ ).

The relative (percent) difference between the proportionate target population values, from data reported by the national immunization programmes and those from the UNPD, is shown for each country in Table 1. For births, the percent difference (absolute value) in the proportionate target population values ranged from $0.4 \%$ (Sao Tome and Principe) to 38.1\% (Namibia) with a median value of 9.7\% (IQR: 3.2\% - 16.9\%). The percent difference in proportionate target population for births, comparing values from reported data to those from UNPD estimates, was $<10 \%$ in 24 (51\%) of 47 countries (difference $<0: 8$ countries; difference $>0: 16$ ), $10 \%-19.9 \%$ in 16 (34\%) countries (difference $<0: 3$; difference $>0: 13$ ) and $20 \%$ or more in seven(15\%) countries (difference $<$ $0: 1$; difference $>0: 6$ ). For surviving infants, the percent difference (absolute value) ranged from $0.4 \%$ (United Republic of Tanzania) to 36.2\% (Namibia) with a median value of $8.5 \%$ (IQR: $2.9 \%$ - 13.2\%). The percent difference in proportionate target population values for surviving infants was $<10 \%$ in 29 (62\%) of 47 countries (difference $<0: 8$ countries; difference $>0: 21$ ), $10 \%-19.9 \%$ in 13 (28\%) countries (difference $<0: 5$; difference $>0: 8$ ) and $20 \%$ or more in five (11\%) countries (difference $<0: 0$; difference $<0: 5$ ). Finally for children under 5 years, the percent difference (absolute value) ranged from $0.9 \%$ (Rwanda) to $44.6 \%$ (Sierra Leone) with 
Table 1. Total population estimates and the number of births, surviving infants (SI) and children under 5 years (U5y) as a proportion of total population for 2013 from the United Nations Population Division and national immunization programmes as reported to the World Health Organization and United Nations Children’s Fund for 47 sub-Saharan African countries.

\begin{tabular}{|c|c|c|c|c|c|c|c|c|c|c|c|}
\hline \multirow{3}{*}{ Country } & \multicolumn{4}{|c|}{$\begin{array}{l}\text { UN Population Division } \\
\text { (UNPD) Estimates }\end{array}$} & \multicolumn{4}{|c|}{$\begin{array}{c}\text { National Immunization Programme } \\
\text { Estimates Reported to WHO } \\
\text { and UNICEF }\end{array}$} & \multirow{2}{*}{\multicolumn{3}{|c|}{$\begin{array}{l}\text { Percent difference in } \\
\text { reported proportionate } \\
\text { target population estimates } \\
\text { compared to UNPD }\end{array}$}} \\
\hline & \multirow{2}{*}{$\begin{array}{l}\text { Total } \\
\text { pop’n } \\
\text { (x1000) }\end{array}$} & \multicolumn{3}{|c|}{ Proportion of total pop'n } & \multirow{2}{*}{$\begin{array}{l}\text { Total } \\
\text { pop'n } \\
\text { (x1000) }\end{array}$} & \multicolumn{3}{|c|}{ Proportion of total pop’n } & & & \\
\hline & & Births & SI & U5y & & Births & SI & U5y & Births & SI & U5y \\
\hline Angola & 21,472 & 0.0442 & 0.0400 & 0.1873 & 22,096 & 0.0500 & 0.0425 & 0.2000 & 13.1 & 6.3 & 6.8 \\
\hline Benin & 10,323 & 0.0365 & 0.0340 & 0.1605 & 9672 & 0.0412 & 0.0384 & 0.1772 & 13.0 & 13.2 & 10.4 \\
\hline Botswana & 2021 & 0.0236 & 0.0228 & 0.1146 & 2025 & 0.0240 & 0.0230 & 0.1216 & 2.0 & 0.8 & 6.1 \\
\hline Burkina Faso & 16,935 & 0.0409 & 0.0381 & 0.1761 & 17,323 & 0.0437 & 0.0407 & 0.1874 & 6.9 & 6.8 & 6.4 \\
\hline Burundi & 10,163 & 0.0448 & 0.0409 & 0.1866 & 8054 & 0.0529 & 0.0417 & 0.2015 & 18.2 & 1.8 & 8.0 \\
\hline Cameroon & 22,254 & 0.0373 & 0.0346 & 0.1631 & 20,896 & 0.0450 & 0.0380 & 0.1690 & 20.6 & 9.8 & 3.6 \\
\hline Cabo Verde & 499 & 0.0202 & 0.0199 & 0.0971 & 512 & 0.0206 & 0.0202 & 0.0981 & 2.1 & 1.8 & 1.1 \\
\hline Central African Republic & 4616 & 0.0342 & 0.0311 & 0.1460 & 4,758 & 0.0350 & 0.0304 & 0.1730 & 2.2 & -2.2 & 18.5 \\
\hline Chad & 12,825 & 0.0460 & 0.0416 & 0.1915 & 13,770 & 0.0410 & 0.0360 & 0.1820 & -10.8 & -13.5 & -5.0 \\
\hline Comoros & 735 & 0.0352 & 0.0329 & 0.1582 & 764 & 0.0267 & 0.0267 & 0.1600 & -24.2 & -18.8 & 1.1 \\
\hline Congo & 4448 & 0.0375 & 0.0352 & 0.1660 & 4413 & 0.0432 & 0.0400 & 0.2000 & 15.1 & 13.6 & 20.5 \\
\hline Côte d'Ivoire & 20,316 & 0.0367 & 0.0339 & 0.1573 & 23,784 & 0.0362 & 0.0331 & 0.1540 & -1.2 & -2.5 & -2.1 \\
\hline $\begin{array}{l}\text { Democratic Republic of the } \\
\text { Congo }\end{array}$ & 67,514 & 0.0428 & 0.0382 & 0.1764 & 86,453 & 0.0400 & 0.0349 & 0.1890 & -6.5 & -8.6 & 7.2 \\
\hline Equatorial Guinea & 757 & 0.0354 & 0.0323 & 0.1517 & 699 & 0.0390 & 0.0354 & 0.1580 & 10.1 & 9.5 & 4.1 \\
\hline Eritrea & 6333 & 0.0367 & 0.0352 & 0.1674 & 3816 & 0.0300 & 0.0287 & 0.1461 & -18.3 & -18.7 & -12.8 \\
\hline Ethiopia & 94,101 & 0.0331 & 0.0315 & 0.1514 & 86,691 & 0.0338 & 0.0316 & 0.1450 & 2.2 & 0.5 & -4.2 \\
\hline Gabon & 1672 & 0.0319 & 0.0305 & 0.1455 & 2008 & 0.0350 & 0.0329 & 0.1550 & 9.7 & 7.7 & 6.5 \\
\hline Gambia & 1849 & 0.0428 & 0.0405 & 0.1832 & 1700 & 0.0481 & 0.0446 & 0.2391 & 12.4 & 10.1 & 30.5 \\
\hline Ghana & 25,905 & 0.0309 & 0.0293 & 0.1419 & 26,594 & 0.0400 & 0.0380 & 0.2000 & 29.6 & 29.7 & 40.9 \\
\hline Guinea & 11,745 & 0.0369 & 0.0343 & 0.1606 & 11,814 & 0.0400 & 0.0360 & 0.2000 & 8.3 & 5.1 & 24.5 \\
\hline Guinea-Bissau & 1704 & 0.0375 & 0.0340 & 0.1583 & 1693 & 0.0370 & 0.0310 & 0.1700 & -1.4 & -8.9 & 7.4 \\
\hline Kenya & 44,354 & 0.0349 & 0.0332 & 0.1589 & 42,436 & 0.0381 & 0.0341 & 0.1604 & 9.1 & 2.8 & 0.9 \\
\hline Lesotho & 2074 & 0.0275 & 0.0259 & 0.1261 & 1909 & 0.0283 & 0.0274 & 0.1369 & 2.9 & 6.1 & 8.5 \\
\hline Liberia & 4294 & 0.0354 & 0.0333 & 0.1598 & 3857 & 0.0430 & 0.0401 & 0.1700 & 21.5 & 20.5 & 6.4 \\
\hline Madagascar & 22,925 & 0.0348 & 0.0335 & 0.1573 & 23,506 & 0.0353 & 0.0333 & 0.1800 & 1.6 & -0.8 & 14.4 \\
\hline Malawi & 16,363 & 0.0398 & 0.0364 & 0.1754 & 15,317 & 0.0437 & 0.0410 & 0.1735 & 9.6 & 12.5 & -1.1 \\
\hline Mali & 15,302 & 0.0473 & 0.0432 & 0.1928 & 16,807 & 0.0443 & 0.0401 & 0.2000 & -6.2 & -7.2 & 3.7 \\
\hline Mauritania & 3890 & 0.0341 & 0.0317 & 0.1502 & 3414 & 0.0440 & 0.0406 & 0.1800 & 29.1 & 28.3 & 19.8 \\
\hline Mauritius & 1244 & 0.0115 & 0.0113 & 0.0564 & 1296 & 0.0104 & 0.0104 & 0.0567 & -9.0 & -7.9 & 0.6 \\
\hline Mozambique & 25,834 & 0.0389 & 0.0361 & 0.1703 & 24,366 & 0.0430 & 0.0402 & 0.1710 & 10.5 & 11.6 & 0.4 \\
\hline Namibia & 2303 & 0.0261 & 0.0252 & 0.1234 & 2173 & 0.0360 & 0.0343 & 0.1400 & 38.1 & 36.2 & 13.4 \\
\hline
\end{tabular}




\begin{tabular}{|c|c|c|c|c|c|c|c|c|c|c|c|}
\hline \multicolumn{12}{|l|}{ Continued } \\
\hline Niger & 17,831 & 0.0499 & 0.0473 & 0.2072 & 16,838 & 0.0583 & 0.0536 & 0.1879 & 16.9 & 13.4 & -9.3 \\
\hline Nigeria & 173,615 & 0.0413 & 0.0382 & 0.1759 & 175,075 & 0.0400 & 0.0376 & 0.1993 & -3.2 & -1.6 & 13.3 \\
\hline Rwanda & 11,777 & 0.0352 & 0.0335 & 0.1648 & 10,811 & 0.0310 & 0.0295 & 0.1650 & -11.9 & -12.0 & 0.1 \\
\hline Sao Tome and Principe & 193 & 0.0339 & 0.0324 & 0.1591 & 173 & 0.0340 & 0.0330 & 0.1500 & 0.4 & 1.8 & -5.7 \\
\hline Senegal & 14,133 & 0.0378 & 0.0360 & 0.1683 & 13,575 & 0.0390 & 0.0390 & 0.1940 & 3.2 & 8.5 & 15.3 \\
\hline Seychelles & 93 & 0.0165 & 0.0164 & 0.0769 & 90 & 0.0175 & 0.0179 & 0.0824 & 6.0 & 9.2 & 7.1 \\
\hline Sierra Leone & 6092 & 0.0366 & 0.0324 & 0.1535 & 6190 & 0.0400 & 0.0364 & 0.2220 & 9.1 & 12.4 & 44.6 \\
\hline Somalia & 10,496 & 0.0439 & 0.0404 & 0.1865 & 9561 & 0.0400 & 0.0357 & 0.2000 & -8.9 & -11.8 & 7.3 \\
\hline South Africa & 52,776 & 0.0208 & 0.0200 & 0.1030 & 52,982 & 0.0205 & 0.0205 & 0.0999 & -1.6 & 2.3 & -3.0 \\
\hline South Sudan & 11,296 & 0.0360 & 0.0332 & 0.1581 & 11,196 & 0.0400 & 0.0359 & 0.2100 & 11.2 & 8.1 & 32.9 \\
\hline Swaziland & 1250 & 0.0298 & 0.0279 & 0.1364 & 1100 & 0.0357 & 0.0288 & 0.1302 & 19.6 & 3.1 & -4.5 \\
\hline Togo & 6817 & 0.0364 & 0.0340 & 0.1604 & 6614 & 0.0450 & 0.0415 & 0.2000 & 23.5 & 21.9 & 24.9 \\
\hline Uganda & 37,579 & 0.0433 & 0.0408 & 0.1893 & 35,189 & 0.0485 & 0.0430 & 0.2050 & 12.1 & 5.3 & 8.3 \\
\hline United Republic of Tanzania & 49,253 & 0.0392 & 0.0373 & 0.1758 & 47,163 & 0.0395 & 0.0375 & 0.1761 & 0.7 & 0.4 & 0.2 \\
\hline Zambia & 14,539 & 0.0430 & 0.0402 & 0.1827 & 14,204 & 0.0500 & 0.0465 & 0.2000 & 16.3 & 15.6 & 9.5 \\
\hline Zimbabwe & 14,150 & 0.0315 & 0.0303 & 0.1443 & 13,205 & 0.0369 & 0.0328 & 0.1514 & 17.0 & 8.2 & 4.9 \\
\hline
\end{tabular}

UNPD data obtained from United Nations, Department of Economic and Social Affairs, Population Division (2013). World Population Prospects: The 2012 Revision.

a median value of 7.1\% (IQR: 3.7\% - 13.4\%). The percent difference in proportionate target population values for children under 5 years was $<10 \%$ in 32 (68\%) of 47 countries (difference $<0: 8$ countries; difference $>0: 24$ ), $10 \%-19.9 \%$ in eight $(17 \%)$ countries (difference $<0: 1$; difference $>0: 7$ ) and $20 \%$ or more in seven (15\%) countries (difference $<0: 0$; difference $>0: 7$ ). The percent difference in proportionate target population was $<10 \%$ for births, surviving infants and children under 5 years in 16 of 47 countries.

\subsection{Re-Computed Target Population Estimates}

We recomputed the estimated number of births, surviving infants and children under 5 years for each country using the proportionate values from the UNPD data and the total population values reported by the national immunization programmes (Table 2). We also display the expected directional change (increase or decrease) in coverage levels if coverage were calculated using a recomputed target population values shown in Table 2 compared to those reported to WHO and UNICEF for a given number of children vaccinated. For example, if a country reported vaccinating 90 children in a target population of 100 children, then the resultant coverage level is $90 \%$. Using the same numerator (e.g., 90 children vaccinated)and re-computing the target population, if the target population size increases compared to the original report, then expected coverage level would decrease and vice versa. Figure 2 displays a comparison of reported administrative coverage levels for the third dose of DTP containing vaccine with those based on a recomputed target population using the UNPD-based proportionate target population value for surviving infants applied to the national immunization programme reported total population. Keep in mind if there was no difference between the compared coverage levels, data points would fall along the 45-degree diagonal.

\subsection{Implications for Immunization Coverage Levels}

Reported administrative $\mathrm{DTP}_{3}$ coverage levels were $<50 \%$ in 3 countries, $50 \%-79 \%$ in 9 countries, $80 \%-89 \%$ in 11 countries, $90 \%$ - 99\% in 19 countries, and $>100 \%$ in five countries. The utilization of the recomputed 
Table 2. Number of births, surviving infants (SI) and children under 5 years (U5y) recomputed using the respective proportion of total population from the United Nations Population Division applied to the national immunization programme reported total population estimate for 2013 for 47 sub-Saharan African countries.

\begin{tabular}{|c|c|c|c|c|c|c|}
\hline \multirow[b]{3}{*}{ Angola } & \multicolumn{6}{|c|}{ Recomputed estimates of number of: } \\
\hline & \multicolumn{2}{|c|}{ Births } & \multicolumn{2}{|c|}{ SI } & \multicolumn{2}{|c|}{ U5y } \\
\hline & 976,547 & $\uparrow$ & 883,571 & $\uparrow$ & $4,137,651$ & $\uparrow$ \\
\hline Benin & 352,595 & $\uparrow$ & 328,584 & $\uparrow$ & $1,552,255$ & $\uparrow$ \\
\hline Botswana & 47,749 & $\uparrow$ & 46,268 & $\uparrow$ & 231,983 & $\uparrow$ \\
\hline Burkina Faso & 708,871 & $\uparrow$ & 660,036 & $\uparrow$ & $3,051,376$ & $\uparrow$ \\
\hline Burundi & 360,516 & $\uparrow$ & 329,418 & $\uparrow$ & $1,503,115$ & $\uparrow$ \\
\hline Cameroon & 779,960 & $\uparrow$ & 723,208 & $\uparrow$ & $3,408,412$ & $\uparrow$ \\
\hline Cabo Verde & 10,343 & $\uparrow$ & 10,170 & $\uparrow$ & 49,741 & $\uparrow$ \\
\hline Central African Republic & 162,894 & $\uparrow$ & 147,847 & $\downarrow$ & 694,521 & $\uparrow$ \\
\hline Chad & 633,229 & $\downarrow$ & 573,056 & $\downarrow$ & $2,637,457$ & $\downarrow$ \\
\hline Comoros & 26,926 & $\downarrow$ & 25,131 & $\downarrow$ & 120,853 & $\uparrow$ \\
\hline Congo & 165,618 & $\uparrow$ & 155,199 & $\uparrow$ & 732,643 & $\uparrow$ \\
\hline Côte d'Ivoire & 872,086 & $\downarrow$ & 807,265 & $\downarrow$ & $3,741,484$ & $\downarrow$ \\
\hline Democratic Republic of the Congo & $3,699,239$ & $\downarrow$ & $3,299,752$ & $\downarrow$ & $15,246,887$ & $\uparrow$ \\
\hline Equatorial Guinea & 24,764 & $\uparrow$ & 22,594 & $\uparrow$ & 106,067 & $\uparrow$ \\
\hline Eritrea & 140,177 & $\downarrow$ & 134,447 & $\downarrow$ & 638,844 & $\downarrow$ \\
\hline Ethiopia & 2,868,335 & $\uparrow$ & $2,728,725$ & $\uparrow$ & $13,126,992$ & $\downarrow$ \\
\hline Gabon & 64,089 & $\uparrow$ & 61,341 & $\uparrow$ & 292,218 & $\uparrow$ \\
\hline Gambia & 72,826 & $\uparrow$ & 68,825 & $\uparrow$ & 311,521 & $\uparrow$ \\
\hline Ghana & 820,836 & $\uparrow$ & 779,248 & $\uparrow$ & $3,774,773$ & $\uparrow$ \\
\hline Guinea & 436,447 & $\uparrow$ & 404,749 & $\uparrow$ & $1,897,234$ & $\uparrow$ \\
\hline Guinea-Bissau & 63,555 & $\downarrow$ & 57,635 & $\downarrow$ & 268,011 & $\uparrow$ \\
\hline Kenya & $1,482,514$ & $\uparrow$ & $1,406,939$ & $\uparrow$ & $6,743,230$ & $\uparrow$ \\
\hline Lesotho & 52,473 & $\uparrow$ & 49,364 & $\uparrow$ & 240,833 & $\uparrow$ \\
\hline Liberia & 136,567 & $\uparrow$ & 128,408 & $\uparrow$ & 616,305 & $\uparrow$ \\
\hline Madagascar & 817,059 & $\uparrow$ & 787,792 & $\downarrow$ & $3,697,724$ & $\uparrow$ \\
\hline Malawi & 610,036 & $\uparrow$ & 558,111 & $\uparrow$ & $2,686,616$ & $\downarrow$ \\
\hline Mali & 794,449 & $\downarrow$ & 726,331 & $\downarrow$ & 3,240,995 & $\uparrow$ \\
\hline Mauritania & 116,391 & $\uparrow$ & 108,086 & $\uparrow$ & 512,774 & $\uparrow$ \\
\hline Mauritius & 14,855 & $\downarrow$ & 14,686 & $\downarrow$ & 73,022 & $\uparrow$ \\
\hline Mozambique & 948,366 & $\uparrow$ & 878,855 & $\uparrow$ & $4,149,057$ & $\uparrow$ \\
\hline Namibia & 56,632 & $\uparrow$ & 54,768 & $\uparrow$ & 268,170 & $\uparrow$ \\
\hline Niger & 840,041 & $\uparrow$ & 795,971 & $\uparrow$ & $3,489,604$ & $\downarrow$ \\
\hline Nigeria & $7,233,313$ & $\downarrow$ & $6,690,486$ & $\downarrow$ & $30,803,031$ & $\uparrow$ \\
\hline
\end{tabular}




\section{Continued}

\begin{tabular}{|c|c|c|c|c|c|c|}
\hline Rwanda & 380,314 & $\downarrow$ & 361,911 & $\downarrow$ & $1,782,207$ & $\uparrow$ \\
\hline Sao Tome and Principe & 5877 & $\uparrow$ & 5,624 & $\uparrow$ & 27,597 & $\downarrow$ \\
\hline Senegal & 513,180 & $\uparrow$ & 488,153 & $\uparrow$ & $2,285,072$ & $\uparrow$ \\
\hline Seychelles & 1488 & $\uparrow$ & 1477 & $\uparrow$ & 6914 & $\uparrow$ \\
\hline Sierra Leone & 226,860 & $\uparrow$ & 200,640 & $\uparrow$ & 950,252 & $\uparrow$ \\
\hline Somalia & 419,632 & $\downarrow$ & 386,654 & $\downarrow$ & $1,782,658$ & $\uparrow$ \\
\hline South Africa & $1,103,100$ & $\downarrow$ & $1,061,102$ & $\uparrow$ & $5,458,027$ & $\downarrow$ \\
\hline South Sudan & 402,827 & $\uparrow$ & 371,891 & $\uparrow$ & $1,769,703$ & $\uparrow$ \\
\hline Swaziland & 32,808 & $\uparrow$ & 30,710 & $\uparrow$ & 150,022 & $\downarrow$ \\
\hline Togo & 240,947 & $\uparrow$ & 225,090 & $\uparrow$ & $1,061,007$ & $\uparrow$ \\
\hline Uganda & $1,522,289$ & $\uparrow$ & $1,436,760$ & $\uparrow$ & $6,662,578$ & $\uparrow$ \\
\hline United Republic of Tanzania & $1,849,077$ & $\uparrow$ & $1,760,365$ & $\uparrow$ & $8,289,549$ & $\uparrow$ \\
\hline Zambia & 610,528 & $\uparrow$ & 571,206 & $\uparrow$ & $2,595,214$ & $\uparrow$ \\
\hline Zimbabwe & 415,798 & $\uparrow$ & 400,479 & $\uparrow$ & $1,906,029$ & $\uparrow$ \\
\hline
\end{tabular}

Arrows indicate the expected directional change, either increase $(\uparrow)$ or decrease $(\downarrow)$, in coverage levels for a given number of children vaccinated due to a change in the target population size based on the application of the proportionate population size from the UNPD estimates applied to the total population reported by the national immunization programme as compared to the estimated target population size reported by national immunization programmes.

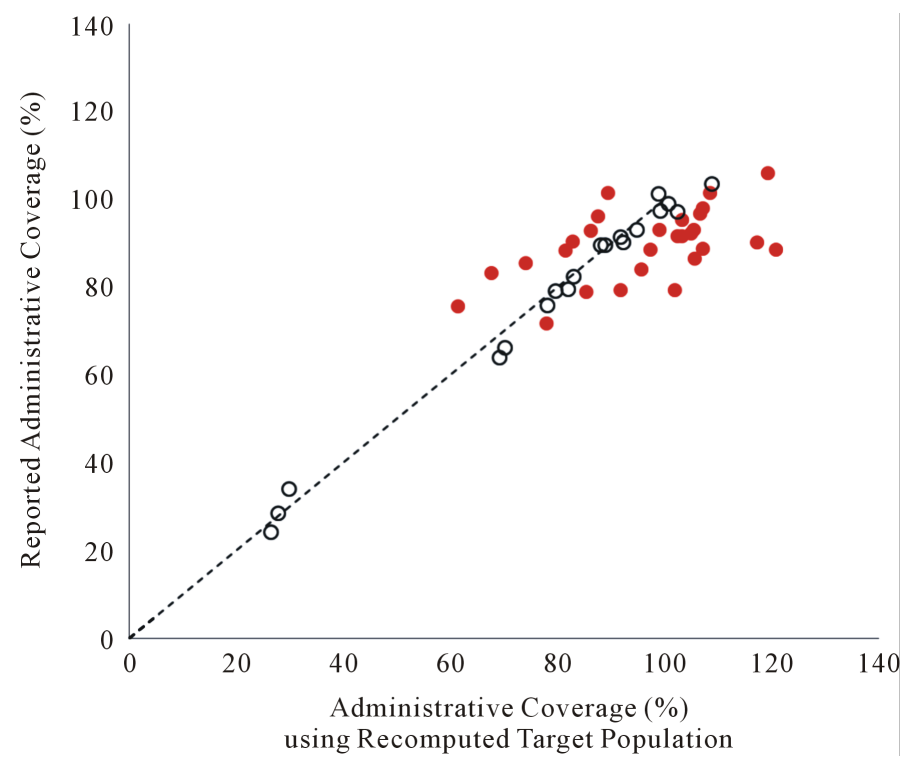

Figure 2. Comparison of administrative coverage for the third dose of DTP containing vaccine based on a recomputed target population using the proportionate target population value from the UNPD applied to the national immunization programme reported total population with reported administrative coverage in 47 sub-Saharan Africa countries for 2013.

target population of surviving infants resulted in lower coverage levels in 13 countries while coverage levels increased in 33 countries, with 17 countries having coverage levels $>100 \%$. Coverage did not meaningfully change in one country. The difference between the reported administrative coverage and the recomputed cover- 
age exceeded 5\% points in 28 of 47 countries (shown in red in Figure 2).

\section{Discussion}

National estimates of births, surviving infants and children under 5 years of age as a proportion of the total national population vary widely across sub-Saharan African countries. We are unaware of any prior work that has described and compared proportionate target population estimates used by immunization programmes to an external source. At the beginning of the Expanded Programme on Immunization in 1974, births and surviving infants as a proportion of the total population in sub-Saharan Africa countries averaged $4.72 \%$ and $4.11 \%$, respectively. By 2013, these values declined to 3.78\% and 3.52\% for sub-Saharan African countries. The national immunization programme in several countries (appear to) continue to use fixed proportionate target population values (e.g., births $=4 \%$ of total population in DRC, Ghana, Guinea, Nigeria, Sierra Leone, Somalia and South Sudan; births $=5 \%$ of total population in Angola and Zambia; children under 5 years $=20 \%$ of total population in Angola, Congo, Ghana, Guinea, Mali, Somalia, South Sudan, Togo, Zambia). Not only can the use of constant proportionate target population values over time present challenges for immunization programme monitoring [3], but inaccuracies in the magnitude of the proportionate target population used may create problems for programme planning.

Comparisons of target proportionate population size based on data reported by national immunization programmes to WHO and UNICEF with values computed from UNPD estimates observed a tendency for immunization programme estimates to be larger than UNPD estimates. Among the five largest countries (based on total population), differences in reported number of births and surviving infants compared to values based on multiplying proportionate population values from the UNPD to nationally reported total population were $<10 \%$. Although the application of the proportionate target population values from the UNPD to the national immunization programme reported national total population reduces the variance to that observed between the nationally reported total population and the UNPD total population, the implication for many countries if they were to use these values would be an implausible immunization coverage level that exceeds $100 \%$. Only three of the 17 countries with recomputed administrative coverage levels $>100 \%$ shown in Figure 2 actually reported a coverage value $>100 \%$. This may suggest problems with numerator data and highlight the need for equally appropriate attention to critically assessing the accuracy of the number of children vaccinated.

The availability of external sources of population data, such as that from the United Nations Population Division, does not represent a solution for inaccuracies that may exist in the national population estimates available to immunization programmes, but they can serve as a useful tool for assessing those inaccuracies and provide useful information for establishing operational envelopes or signals for further investigation. Ideally, immunization programme staff work closely with their technical counterparts in the national statistics system to make use of the latest census data and population projections in order to obtain estimates of births and surviving infants that are more appropriate than using a constant proportionate target population value over time. Anecdotally we know that this is often not the case.

Given the dramatic changes in fertility and in neonatal infant and under-5 mortality since the beginning of the Expanded Programme on Immunization, as well as expected changes between now and mid-century, national immunization programmes confront an on-going challenge to effectively plan the delivery of immunization services, manage stock and supply levels and target interventions. This is particularly so for the programmes in sub-Saharan Africa, which will account for one in every three children born by 2050 [4]. Because childhood immunization-related target populations are among the more difficult ones to accurately estimate, immunization programmes in sub-Saharan Africa are encouraged to include a critical assessment of target population values, in conjunction with the national statistics system, as part of the on-going programme monitoring process. In addition, consideration may be given to include training exercises in such assessments within mid-level programme manager continuing education workshops. Further work is needed to describe different approaches that have proved successful in fostering technical collaborations between national immunization programmes and their counterparts in national statistical systems.

\section{Disclaimer}

The findings and opinions expressed here are those of the authors alone and do not reflect those of their respective institutions. 


\section{References}

[1] (2010) United Nations Department of Economic and Social Affairs, 2010 World Population and Housing Census Programme: Census Dates for All Countries. http://unstats.un.org/unsd/demographic/sources/census/censusdates.htm

[2] United Nations, Department of Economic and Social Affairs, Population Division (2013) World Population Prospects: The 2012 Revision. UNDESA, New York.

[3] Brown, D.W., Feeney, G. and Burton, A.H. (2012) Raising Awareness among Immunization Programme Managers to the Potential Bias Resulting from the Application of Fixed Factors to Obtain Target Population Size Estimates. The Open Public Health Journal, 5, 15-18. http://www.benthamscience.com/open/tophj/articles/V005/15TOPHJ.pdf

[4] (2012) United Nations Children’s Fund (UNICEF) Generation 2025 and Beyond. The Critical Importance of Understanding Demographic Trends for Children of the 21st Century. UNICEF, New York. http://www.childinfo.org/files/Generation 2025 and beyond Nov2012.pdf 
Scientific Research Publishing (SCIRP) is one of the largest Open Access journal publishers. It is currently publishing more than 200 open access, online, peer-reviewed journals covering a wide range of academic disciplines. SCIRP serves the worldwide academic communities and contributes to the progress and application of science with its publication.

Other selected journals from SCIRP are listed as below. Submit your manuscript to us via either submit@scirp.org or Online Submission Portal.
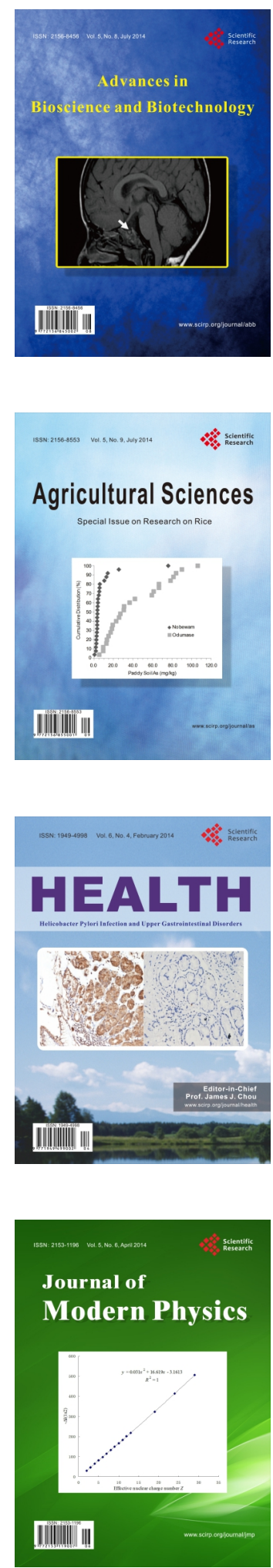
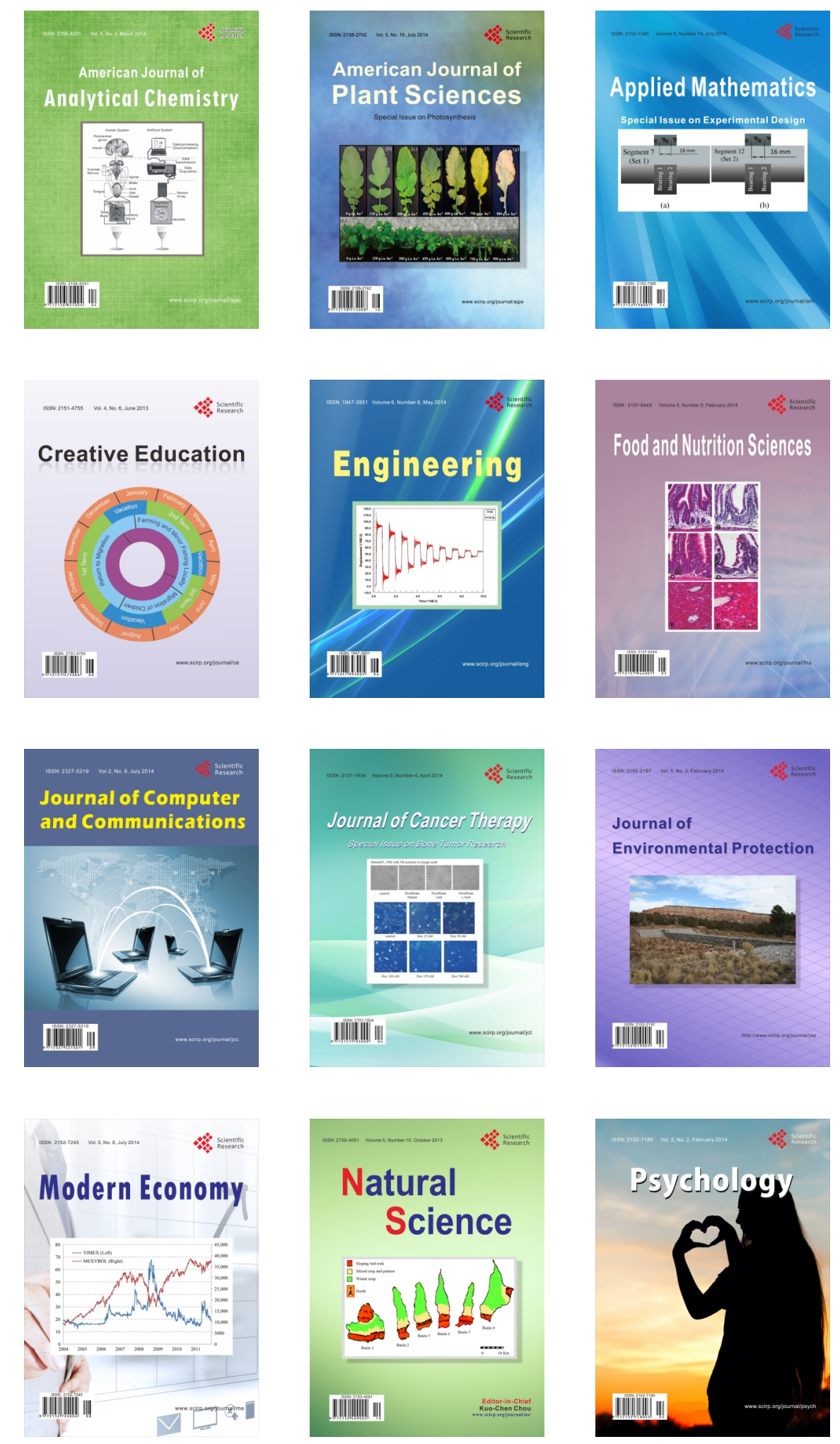